\title{
Congenital Hypothyroidism due to Mutations in the Sodium/lodide Symporter Identification of a Nonsense Mutation Producing a Downstream Cryptic 3' Splice Site
}

\author{
Joachim Pohlenz, ${ }^{\ddagger}$ Ira M. Rosenthal, ${ }^{\ddagger}$ Roy E. Weiss, ${ }^{\star}$ Sissy M. Jhiang,,${ }^{\mid \uparrow}$ Charles Burant, ${ }^{\star}$ and Samuel Refetoff ${ }^{\star \ddagger \S}$ \\ $*$ Department of Medicine, ${ }^{\ddagger}$ Department of Pediatrics, and ${ }^{\S}$ The J.P. Kennedy, Jr., Mental Retardation Research Center, The University of \\ Chicago, Chicago, Illinois 60637-1470; and $\|^{\|}$epartment of Physiology and ${ }^{\mathbb{I}}$ Department of Internal Medicine, The Ohio State University, \\ Columbus, $\mathrm{OH} 43210$
}

\begin{abstract}
A 12-yr-old hypothyroid girl was diagnosed at birth as athyreotic because her thyroid gland could not be visualized by isotope scanning. Goiter development due to incomplete thyrotropin suppression, a thyroidal radioiodide uptake of $<1 \%$, and a low saliva to plasma ratio of 2.5 suggested iodide $\left(\mathrm{I}^{-}\right)$transport defect. mRNA isolated from her thyroid gland and injected into Xenopus oocytes failed to increase $\mathrm{I}^{-}$transport.

Sequencing of the entire $\mathrm{Na}^{+} / \mathrm{I}^{-}$symporter (NIS) cDNA revealed a $C$ to $G$ transversion of nucleotide (nt) 1146 in exon 6, resulting in a Gln 267 (CAG) to Glu (GAG) substitution. This missense mutation produces an NIS with undetectable $\mathrm{I}^{-}$transport activity when expressed in COS-7 cells. Although only this missense mutation was identified in thyroid and lymphocyte cDNA, genotyping revealed that the proposita and her unaffected brother and father were heterozygous for this mutation. However, amplification of cDNA with a primer specific for the wild-type nt 1146 yielded a sequence lacking $67 \mathrm{nt}$. Genomic DNA showed a

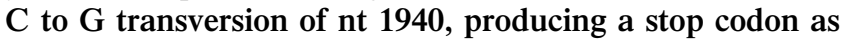
well as a new downstream cryptic $3^{\prime}$ splice acceptor site in exon 13, responsible for the $67 \mathrm{nt}$ deletion, frameshift, and premature stop predicting an NIS lacking 129 carboxy-terminal amino acids. This mutation was inherited from the mother and present in the unaffected sister.

Thus, although the proposita is a compound heterozygote, because of the very low expression $(<2.5 \%)$ of one mutant allele, she is functionally hemizygous for an NIS without detectable bioactivity. (J. Clin. Invest. 1998. 101: 1028-1035.) Key words: frameshift • genetics • thyroid • iodide transport • allele-specific amplification
\end{abstract}

\section{Introduction}

Iodine is indispensable for the biosynthesis of thyroid hormone. Under normal conditions of dietary iodine intake, a suf-

Presented in part at the 70th annual meeting of the American Thyroid Association, Colorado Springs, CO, 15-17 October 1997.

Address correspondence to Samuel Refetoff, M.D., The University of Chicago, 5841 South Maryland Avenue, Chicago, IL 60637. Phone: 773-702-6939; FAX: 773-702-6940; E-mail: refetoff@medicine. uchicago.bsd.edu

Received for publication 14 August 1997 and accepted in revised form 19 December 1997.

J. Clin. Invest.

(C) The American Society for Clinical Investigation, Inc. 0021-9738/98/03/1028/08 \$2.00

Volume 101, Number 5, March 1998, 1028-1035

http://www.jci.org ficient supply of iodine to the thyroid gland requires active iodide $\left(\mathrm{I}^{-}\right)$uptake into thyroid follicular cells, a process which is mediated by the sodium/iodide symporter (NIS) ${ }^{1}(1)$. This protein with a predicted molecular mass of $\sim 65 \mathrm{kD}$ belongs to the family of sodium-dependent cotransporters and has most sequence similarity with the human sodium/glucose cotransporter 1 (SGLT1). The NIS is located in the basolateral membrane of the thyroid follicular cell and is predicted to have a serpentine structure of probably 12 transmembrane segments, with both carboxy and amino termini located inside the cell (for a review, see reference 1). The hypothyroidism due to abnormalities of the NIS can be overcome by the supplementation of excess $\mathrm{I}^{-}$as well as with levothyroxine (L- $\left.\mathrm{T}_{4}\right)(2)$.

Routine neonatal screening in most western countries has shown an incidence of hypothyroidism of about 1 in 4,000 newborn (3). Congenital hypothyroidism is sporadic in the majority of cases, and is caused by thyroid agenesis or dysgenesis (ectopia or hypoplasia) that appears not to have a genetic basis $(2,4)$. In $\sim 15 \%$ of the cases, hypothyroidism results from defects of thyroid hormonogenesis inherited in an autosomal recessive manner. Such defects have been traced to abnormalities in all known steps involved in thyroid hormone synthesis, including thyrotropin (TSH), TSH receptor, $\mathrm{I}^{-}$accumulation ("trapping"), $\mathrm{I}^{-}$organification, thyroglobulin, and iodotyrosine deiodination $(2,5)$. Homozygous or compound heterozygous mutations have been described in the TSH- $\beta$ (6), TSH receptor (7), thyroid peroxidase (8), and thyroglobulin (9) genes. However, until the very recent publication of Fujiwara et al. (10), the precise gene abnormalities causing a defect in $\mathrm{I}^{-}$trapping were not known.

Since the first report in 1958 of a hypothyroid cretin with a presumed $\mathrm{I}^{-}$trapping defect (11), about 40 additional cases belonging to 28 families and occurring world-wide have been reported (for a review, see reference 2 ). However, determination of the molecular mechanism of this form of congenital hypothyroidism had to await the cloning of the NIS gene. First, the rat NIS cDNA was cloned by Dai et al. in 1996 (12). This was followed in the same year by the cloning of the human NIS cDNA by Smanik et al. (13). The human NIS gene is located on chromosome 19. It consists of 15 exons and encodes a protein of 643 amino acids (14).

Here, we describe a patient with a congenital $\mathrm{I}^{-}$trapping defect who is heterozygous for two different mutations in the NIS gene. The mutant allele inherited from the father, which has a single amino acid substitution (Q267E), is transcribed in thyroid tissue and lymphocytes but has no detectable biological activity. The mutation inherited from the mother produces

1. Abbreviations used in this paper: $\mathrm{L}_{-} \mathrm{T}_{4}$, levothyroxine; NIS, sodium/ iodide symporter; nt, nucleotide(s); SGLT1, sodium/glucose cotransporter 1; S/P, saliva to plasma; TSH, thyrotropin; WT, wild-type. 
a stop codon and also creates a downstream cryptic $3^{\prime}$ splice acceptor site in exon 13 of the NIS gene. The latter results in a 67-bp deletion and a frameshift with a premature stop in the transcript, predicting a truncated NIS lacking 129 amino acids. The mutant NIS mRNA transcribed from the maternal allele is unstable as determined from the very low steady state levels of mRNA. Thus, the proposita is functionally hemizygous for an NIS without detectable bioactivity in vitro.

\section{Methods}

Patient. The proposita, a daughter of unrelated healthy parents of Mexican Hispanic ancestry, had a normal, spontaneous vaginal delivery at term after an unremarkable gestation. Her birth weight was 3.1 $\mathrm{kg}$. Routine neonatal screening for hypothyroidism revealed a blood $\mathrm{TSH}$ of $142 \mathrm{mU} /$ liter $\left(\right.$ normal $<30$ ) and a $\mathrm{T}_{4}$ of $6.7 \mu \mathrm{g} / \mathrm{dl}$ (normal $>7$ ). Serum TSH levels remained high ( $>40 \mathrm{mU} /$ liter) at $3 \mathrm{wk}$ of age. No thyroid gland was found on physical examination and no functioning tissue was detected by pertechnetate $\left.\left({ }^{99 \mathrm{~m}} \mathrm{Tc}\right] \mathrm{ClO}_{4}\right)$ scan in the neck or base of the tongue. From these findings, it was concluded that the infant had thyroid gland agenesis, and treatment with $37.5 \mu \mathrm{g} / \mathrm{d}$ $\mathrm{L}_{-} \mathrm{T}_{4}$ was started. With adjustments of the $\mathrm{L}-\mathrm{T}_{4}$ dose, physical and mental development proceeded normally. The patient grew along the 90th percentile for height and the 95th percentile for weight. Several elevated values of serum TSH were recorded, including $50 \mathrm{mU} /$ liter at $11 \mathrm{mo}$ of age and $15.4 \mathrm{mU} / \mathrm{liter}$ at $7 \mathrm{yr}$ of age. After each of these occurrences, the $\mathrm{L}_{-} \mathrm{T}_{4}$ dose was increased to normalize the level. However, in addition to these high TSH values, of the remaining 23 TSH determinations recorded during $11 \mathrm{yr}$ of follow-up, only 6 were within the normal range while the remainder ranged from 5.8 to 12.7 $\mathrm{mU} /$ liter (normal 0.5-4.5).

At $12 \mathrm{yr}$ of age, the patient noted a mass on the right side of her neck. On physical examination, it was $2.5 \mathrm{~cm}$ in diameter, firm, freely moveable, and nontender; it had not been observed $1 \mathrm{yr}$ earlier. The patient was taking $100 \mu \mathrm{g}$ of $\mathrm{L}_{-} \mathrm{T}_{4}$, and her serum total $\mathrm{T}_{4}$ level was 7.2 $\mu \mathrm{g} / \mathrm{dl}$ (normal range $5.0-12$ ), with a TSH of $14.5 \mathrm{mU} / \mathrm{liter}$ (normal range $0.4-3.6)$. The thyroidal ${ }^{123} \mathrm{I}^{-}$uptake was markedly reduced (3\% at $2 \mathrm{~h}, 2 \%$ at $4 \mathrm{~h}$, and $<1 \%$ at 6 and $24 \mathrm{~h}$ ). Scanning showed faint visualization of both thyroid lobes, with possible reduction of activity in the right lower pole. Ultrasound displayed a very heteroechogenic thyroid gland and confirmed the finding of a solid, highly vascular mass measuring $\sim 3 \mathrm{~cm}$ in diameter in the lower pole of the right lobe. Microscopic examination of cells obtained by fine needle aspiration showed abnormalities suggestive of a dyshormonogenic gland, but follicular carcinoma could not be ruled out. Therefore, the patient underwent near total thyroidectomy. Histological examination revealed a hyperplastic thyroid parenchyma with an encapsulated hyperplastic nodule $2.5 \mathrm{~cm}$ in diameter and several smaller hyperplastic and colloid nodules. As described previously (15), many thyroid epithelial cells had bizarre nuclear pleomorphism as seen in thyroid malignancy, but which were attributed to chronic TSH stimulation.

To confirm the diagnosis of an iodide trapping defect suggested by the clinical, laboratory, and histological findings, the $\mathrm{I}^{-}$saliva to plasma (S/P) ratio was measured $1 \mathrm{~h}$ after the oral administration of $\mathrm{Na}^{125} \mathrm{I}$. This S/P ratio was 2.5 in the patient (normal 25-140). After thyroidectomy and under treatment with $125 \mu \mathrm{g} \mathrm{L}-\mathrm{T}_{4}$, the patient remains euthyroid and is doing well in school.

Family members. Both her parents, her younger brother and a sister, three maternal uncles, a paternal uncle, and a paternal aunt were clinically euthyroid and had normal or slightly enlarged $(<30 \mathrm{~g})$ thyroid glands. All gave informed consent to undergo studies approved by the Institutional Review Board at The University of Chicago.

All had normal S/P ratios ranging from 29 to 70 (Fig. $1 A$ ). Values for two normal controls determined at the same time were 52 and 56. Serum tests of thyroid function including total and free $\mathrm{T}_{4}$, total triiodothyronine, and TSH were in the normal range ( $\mathrm{TSH}$ values are

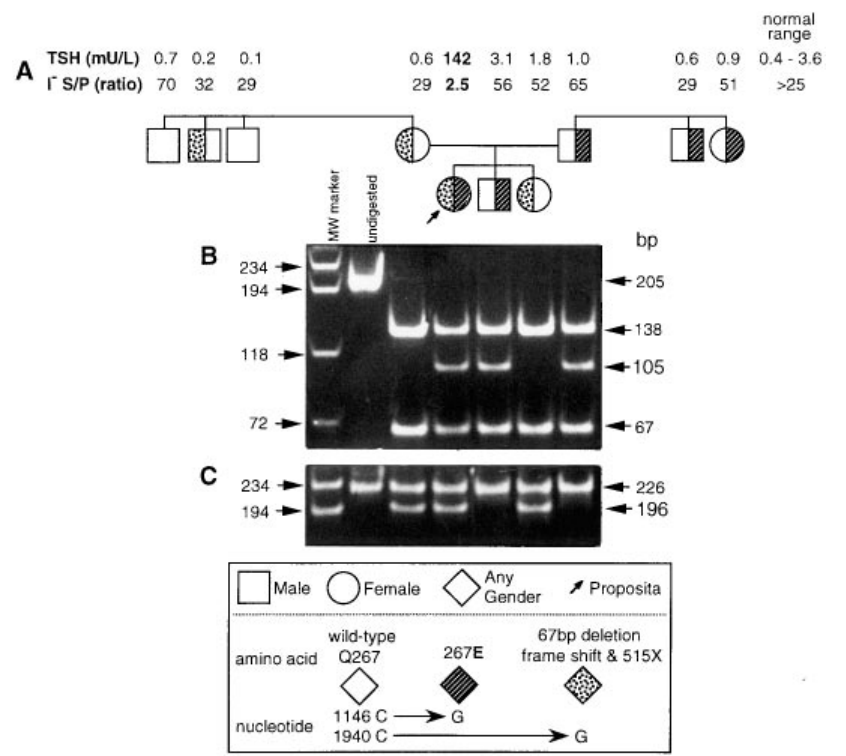

Figure 1. Pedigree, phenotype, and genotype of the proposita and family members. $(A)$ The phenotype is shown in terms of serum TSH levels and $\mathrm{I}^{-} \mathrm{S} / \mathrm{P}$ ratios. TSH values for the proband are those obtained before treatment with $\mathrm{L}_{-} \mathrm{T}_{4}$. $(B)$ Detection of the paternal mutation in nt 1146. A 205-bp fragment was amplified from genomic DNA using a mismatched primer that creates a BsII restriction site in the presence of the mutant nt $1146 \mathrm{G}$. There is also a second, natural BslI site in the adjacent intron, included in the amplified DNA fragment. The product of digestion was resolved by electrophoresis on $10 \%$ polyacrylamide gel. Digestion of the allele containing the WT 1146C produces two fragments, of 138 and $67 \mathrm{bp}$. Digestion of the mutant allele $1146 \mathrm{G}$ produces three fragments, of 105,67 , and $33 \mathrm{bp}$. The 33-bp fragment is not visible on the agarose gel. Note that the proposita is heterozygous for this mutation, showing both the 138and 105-bp fragments. $(C)$ Detection of the maternal mutation in $\mathrm{nt}$ 1940. A 226-bp fragment was amplified from genomic DNA using a mismatched primer that produces a BslI restriction site only in the presence of the mutant nt 1940G. The product of digestion was resolved by electrophoresis on $10 \%$ polyacrylamide gel. The mutant allele produces two fragments, of 196 and $30 \mathrm{bp}$, the latter not visible. Note that the proposita is heterozygous for this mutation, showing both the 226- and 196-bp fragments.

shown in Fig. $1 A$ ). Thyroid peroxidase and thyroglobulin antibodies were not present in any samples including that of the proposita.

Clinical testing. S/P ratio was measured by a modification of the method of Harden et al. (16). Saliva was collected without stimulation over a period of 5-10 $\min 1 \mathrm{~h}$ after the oral administration of 5 $\mu \mathrm{Ci}$ of $\mathrm{Na}^{125}$. At the same time, a venous blood sample was obtained, and the $\mathrm{S} / \mathrm{P}$ ratio of radioiodide was determined by counting equal volumes of these fluids. Serum total $\mathrm{T}_{4}$ and triiodothyronine concentrations were measured by RIA, and TSH levels were determined by a third generation chemiluminescence assay (Corning Nichols Institute, San Juan Capistrano, CA). The serum free $\mathrm{T}_{4}$ index was calculated as the product of the serum total $T_{4}$ and the $T_{4}$ resin uptake value. Thyroid peroxidase and thyroglobulin autoantibodies were measured by agglutination (Fujirebio Inc., Tokyo, Japan).

Preparation of RNA and DNA. RNA was prepared from thyroid tissue and from lymphocytes. Thyroid tissue removed at surgery from the proposita and from patients with multinodular goiter and Graves' disease ("normal thyroid glands") were frozen immediately and kept at $-85^{\circ} \mathrm{C}$. Frozen thyroid tissue $(0.2-2 \mathrm{~g})$ was pulverized in a cold 
Table I. Primers Used for Amplification of cDNA and Sequencing

\begin{tabular}{|c|c|c|}
\hline Primer pair & Sequence & Orientation \\
\hline I & $\begin{array}{l}\text { 5' - AGACGGAGCGGGGACAGGCTGCCGAGCA - } \\
\text { TCCTCCCACCCG - 3' } \\
\text { 5' - GTGGACACCAGGAGCATGAG - 3' }\end{array}$ & $\begin{array}{l}\text { Sense } \\
\text { Antisense }\end{array}$ \\
\hline II & $\begin{array}{l}\text { 5' - ACCTTCGGCGCCTGGGACTA - 3' } \\
5^{\prime} \text { - GATGCCGGTGTACAGCATCG - 3' }\end{array}$ & $\begin{array}{l}\text { Sense } \\
\text { Antisense }\end{array}$ \\
\hline III & $\begin{array}{l}\text { 5' - AAGTTCCTGTGGATGTGC - 3' } \\
5^{\prime}-\text { CACGCCATACATGGAGAGCCAC - 3' }\end{array}$ & $\begin{array}{l}\text { Sense } \\
\text { Antisense }\end{array}$ \\
\hline IV & $\begin{array}{l}\text { 5' - TGCTAAGTGGCTTCTGGGTTGT - 3' } \\
\text { 5' - ATGCTGGTGGATGCTGTGCTGA - 3' }\end{array}$ & $\begin{array}{l}\text { Sense } \\
\text { Antisense }\end{array}$ \\
\hline $\mathrm{V}$ & $\begin{array}{l}5^{\prime} \text { - ATGAATTCCTGATCGTGTCCAGC - 3' } \\
5^{\prime} \text { - GGATCCCGTCCATTCCTGAG - 3' }\end{array}$ & $\begin{array}{l}\text { Sense } \\
\text { Antisense }\end{array}$ \\
\hline VI & $\begin{array}{l}\text { 5' - GAGCCCTTCATCTTGGGAATGT - 3' } \\
\text { 5' - ACAGACGATCCTCATTGGTG - 3' }\end{array}$ & $\begin{array}{l}\text { Sense } \\
\text { Antisense }\end{array}$ \\
\hline VII & $\begin{array}{l}\text { 5' - GTGGCTCTCTCAGTCAACGCCTCT - 3' } \\
\text { 5' - TAGGGTAGGGTATTGTAGTCCT - 3' }\end{array}$ & $\begin{array}{l}\text { Sense } \\
\text { Antisense }\end{array}$ \\
\hline
\end{tabular}

PCR conditions: initial denaturation, $5 \mathrm{~min}$ at $94^{\circ} \mathrm{C} ; 35$ cycles: $1 \mathrm{~min}$ at $94^{\circ} \mathrm{C}, 1 \mathrm{~min}$ at $55^{\circ} \mathrm{C}$ (primer pair I, $60^{\circ} \mathrm{C}$ ), $1 \mathrm{~min}$ at $72^{\circ} \mathrm{C}$ and final extention $10 \mathrm{~min}$ at $72^{\circ} \mathrm{C}$. Mismatched $\mathrm{nt}$ are underlined.

$\left(-85^{\circ} \mathrm{C}\right)$ mortar. Total RNA was extracted using the phenol/guanidine isothiocyanate method $\left(\right.$ TRIzol $^{\circledR}$; GIBCO BRL, Gaithersburg, MD). Lymphocytes from $30 \mathrm{ml}$ of blood were isolated by Ficol Paque Plus $^{\circledR}$ (Pharmacia Biotech, Piscataway, NJ) according to the manufacturer's protocol, and total RNA was extracted using TRIzol. PolyA ${ }^{+}$ RNA from thyroid was isolated using the oligotex mRNA kit (QIAGEN Inc., Chatsworth, CA) and was further fractionated by a $10-30 \%$ sucrose gradient centrifugation. Fractions containing poly $\mathrm{A}^{+}$ RNA of 2-4.5 kb and enriched with NIS mRNA (17) were pooled, and the RNA was ethanol-precipitated and dissolved in water $(1 \mu \mathrm{g} / \mathrm{ml})$. After each step of preparation, the integrity of the RNAs was assessed by formaldehyde-agarose gel electrophoresis. The size-selected mRNA was used for expression studies in Xenopus oocytes while total RNA was used for the preparation of cDNA.

Sequencing of the NIS gene. Both genomic DNA and cDNA amplified by PCR were used for sequencing. DNA was extracted from leukocytes by standard procedure. Avian myeloblastosis virus reverse transcriptase (Promega Corp., Madison, WI) and oligo dT primer were used to prepare the first strand of cDNA from thyroid tissue and lymphocyte total RNA. Oligonucleotide primers for amplification and sequencing of NIS cDNA are shown in Table I. Automated fluorescence-based sequencing (ABI; Perkin-Elmer Corp., Foster City, CA) was performed either directly or after cloning of the PCR products into pGEM-T vector (Promega Corp.).

The weakly expressed mutant allele (see Results) was amplified from the proposita's cDNA using allele-specific oligonucleotide primers with the $3^{\prime}$ terminal nucleotide (nt) complementary for the wild-type (WT) nt $1146 \mathrm{C}$ and thus, mismatched for the mutant nt 1146G (nt numbers are those published for the human NIS cDNA [13]). These primers were tested for their amplification specificity using clones containing either the mutant or WT NIS cDNA as templates, under optimal PCR conditions. A PCR fragment of 961 bp in the 5' direction from nt 1146 was generated using the allele-specific antisense primer 5'-CTTCTCTGTGCGGCAAGCCACGTATCGCTG$3^{\prime}$ (mismatched nt, to increase specificity [18], is underlined, and the allele-specific nt is in bold) and the sense primer 5'-AGACGGAGCGGGGACAGGCTGCCGAGCATCCTCCCACCCG-3' . A PCR fragment of $1061 \mathrm{bp}$ in the $3^{\prime}$ direction of nt $1146 \mathrm{C}$ was generated using the allele-specific sense primer $5^{\prime}$-TGGCGTGAACCAG-
GCGTAGGTCC- $3^{\prime}$ and the antisense primer $5^{\prime}$-ACAGACGATCCTCATTGGTG- $3^{\prime}$. The PCR products were purified and cloned in the pGEM-T, and eight clones in each direction were sequenced.

Genotyping. The mutations in nt 1146 and nt 1940 do not create new restriction sites. Therefore, the endonuclease digestion allelespecific primer method (19) was used to detect these two mutations in genomic DNA. For this purpose, mismatched oligonucleotide primers complimentary to sequences near but not overlapping the mutant nt were synthesized. These primers were designed so that their product of amplification would create a restriction site only if the templates contained the mutant nt.

For detection of the mutation in nt 1146, the sense primer 5'-GTGTGGCTCTCCATGTATGGCGTGAACTACCCGCAG-3' (mismatched nt underlined) and the antisense primer $5^{\prime}$-AGGCCGACCTGGTTGATGAGCAGGGC-3' were used to amplify a 205-bp fragment. The mismatched nt create a BslI restriction site only if the mutant nt $1146 \mathrm{G}$ is present. The PCR fragment also contains a second, natural BsII site in the adjacent intron, as a control. The products of digestion with BsII were resolved by $10 \%$ PAGE and stained with ethidium bromide. The allele containing the WT $1146 \mathrm{C}$ produces two fragments, of 138 and $67 \mathrm{bp}$, whereas the mutant allele $1146 \mathrm{G}$ produces three fragments, of 105,67 , and $33 \mathrm{bp}$.

For detection of the mutation in nt 1940, the mismatched sense primer 5'-GCTGACAGCTTCTATGCCATCTCCTACCTCTATT$3^{\prime}$ and the antisense primer $5^{\prime}$-ggtacaaatgtgtacagcacacagtggcagtttg- $3^{\prime}$ (intron sequences in lower case letters) were used to amplify a 226-bp fragment. The mismatched nt creates a BslI restriction site only in the presence of the mutant nt $1940 \mathrm{G}$. After digestion and resolution by $10 \%$ PAGE, the mutant allele produces two fragments, of 196 and 30 bp, whereas the allele containing the WT nt $1940 \mathrm{C}$ remains undigested.

Construction of plasmids, cell culture, and functional expression. A mammalian expression vector (pcDNA3/Amp; Invitrogen Corp., San Diego, CA) containing the entire cDNA of the human WT NIS was constructed as follows. First, a PCR fragment containing the full coding sequence of the NIS was amplified from a normal thyroid gland cDNA with a sense primer containing a HindIII site (mismatched nt underlined) and the natural Kozak sequence (in bold), 5'-GATGAAGCTTGCCCTCATGGAGGCCGTGGAGACCGGGGAACG $\overline{\mathrm{GCC}} \overline{\mathrm{AC}}-3^{\prime}$, and the antisense primer, 5'-AGGACTACAATACCCTACCCTA-3'. The PCR product was cloned into the pGEM-T vector. The insert was released by HindIII and SacII digestion. After blunting the sticky end of SacII, the fragment was inserted into the HindIII/Eco RV polylinker site of pcDNA3/Amp. Fragments of both mutant NIS cDNAs were amplified by PCR and substituted for the corresponding fragments of WT NIS cDNA in pGEM-T, and the mutant NISs were transferred and cloned into pcDNA3/Amp as described above. All DNA constructs were verified by sequencing. When expressed in reticulocyte lysate (T7-coupled TNT ${ }^{\circledR}$ lysate; Promega Corp.), all constructs gave unique products on SDS-PAGE (data not shown).

COS-7 cells were maintained and propagated in DME containing $10 \%$ FBS (GIBCO BRL) at $37^{\circ} \mathrm{C}$ under $100 \%$ humidity in $90 \%$ room air and $10 \% \mathrm{CO}_{2} .1 \mathrm{~d}$ before transfection, the cells were trypsinized and plated on $10-\mathrm{cm}$ cell culture dishes. When cells reached a density of $70-80 \%$, they were transfected with various combinations of plasmids using the calcium phosphate coprecipitation method (20). These included the WT NIS, the mutant NIS 267E, a combination of both in equal amounts, or the empty pcDNA3/Amp vector. Sonicated salmon sperm DNA was added to maintain the total concentration of transfected DNA constant. A luciferase expression plasmid (20) in a concentration $1 / 10$ of the total DNA was cotransfected to monitor the efficiency of transfection. After $16-20 \mathrm{~h}$ incubation at $37^{\circ} \mathrm{C}$ and $5 \%$ $\mathrm{CO}_{2}$, the transfected cells from each 10-cm dish were trypsinized and distributed uniformly onto 6-well dishes in which the $\mathrm{I}^{-}$uptake studies were carried out under the three different conditions (see below). The similarity of luciferase values indicated that this procedure reduced intraassay variation. The amount of DNA used for transfec- 
tion, which was optimized to give the maximal $\mathrm{I}^{-}$uptake, was $5 \mu \mathrm{g} /$ well.

Expression of NIS mRNA in Xenopus laevis oocytes. Xenopus laevis oocytes were prepared, and $24 \mathrm{~h}$ later, healthy-appearing stage $\mathrm{V}$ or VI oocytes were selected for injection. 20 oocytes were injected with each of the following preparations: water only, or size-selected polyA ${ }^{+}$RNA extracted from thyroid glands of unaffected individuals or the proposita. Each oocyte was injected with $50 \mu \mathrm{l}(1 \mathrm{mg} \mathrm{RNA} / \mathrm{ml})$ near the vegetal hemisphere. After $5 \mathrm{~d}$ of incubation at $20^{\circ} \mathrm{C}$, oocytes were assayed for specific $\mathrm{I}^{-}$uptake as described below. The details of oocyte dissection, handling, injection, and incubation have been described in detail elsewhere (21).

Iodide uptake assay. Iodide transport into Xenopus oocytes (4 d after microinjection) and into COS-7 cells (60 h after transfection) was determined by measurement of specific uptake of ${ }^{125} \mathrm{I}^{-}$essentially as described by Vilijn and Carrasco (17) and Weiss et al. (22), respectively, with slight modifications.

Oocytes were incubated for 60 rather than $45 \mathrm{~min}$, and the solutions contained 5 rather than $50 \mu \mathrm{M}$ of $\mathrm{NaI}$ and $0.5 \mu \mathrm{Ci}$ of ${ }^{125} \mathrm{I}$. Otherwise, the procedure was followed as described (17).

Duplicate wells of COS-7 cells, transfected with each combination of DNA constructs, were washed once with $2 \mathrm{ml}$ of three different modified HBSS solutions, one containing $137 \mathrm{mM} \mathrm{NaCl}$, the second having in addition $10 \mu \mathrm{M}$ sodium perchlorate $\left(\mathrm{NaClO}_{4}\right)$, and the third containing $137 \mathrm{mM}$ choline chloride instead of $\mathrm{NaCl}$. The cells were then incubated for $1 \mathrm{~h}$ at $37^{\circ} \mathrm{C}$ and $5 \% \mathrm{CO}_{2}$ in the respective solutions to which $0.1 \mu \mathrm{Ci}$ carrier-free $\mathrm{Na}^{125} \mathrm{I}$ (ICN Biomedicals, Inc., Irvine, $\mathrm{CA}$ ) and $0.1 \mu \mathrm{M}$ unlabeled $\mathrm{NaI}$ had been added. The medium was aspirated, and to reduce the leakage of $\mathrm{I}^{-}$that had been transported into the cells, the individual wells were washed rapidly one by one with ice-cold HBSS (GIBCO BRL). Cells were harvested after incubation in $200 \mu \mathrm{l}$ cell culture lysis buffer (Promega Corp.) for $5 \mathrm{~min}$. Finally, an aliquot of $20 \mu \mathrm{l}$ was assayed for luciferase activity using luciferase assay reagents (Promega Corp.), and the remaining cell lysate was counted in a $\gamma$-scintillation counter (COBRA; Packard, Meriden, CT). The amount of radioactivity was expressed as counts per minute (cpm) corrected for the efficiency of transfection (each $\mathrm{cpm}$ value was divided by the corresponding luciferase measurement and multiplied by the average luciferase value for a given experiment).

\section{Results}

Expression of NIS mRNA in Xenopus oocytes. Before the availability of the NIS sequence, size-selected (2-4.5 kb) polyA ${ }^{+}$ RNA preparations from the patient's thyroid gland and from thyroid tissue of an unrelated individual with intact $\mathrm{I}^{-}$transport function were expressed in Xenopus oocytes. As shown in Fig. 2, $\mathrm{I}^{-}$uptake inhibited by $\mathrm{ClO}_{4}^{-}$was observed in oocytes injected with the control RNA but not in those injected with RNA prepared from the proposita's thyroid gland. $\mathrm{I}^{-}$uptake of oocytes injected with RNA from the proposita was not greater than that of oocytes injected with water alone. Normal RNA produced a ninefold increase in $\mathrm{I}^{-}$uptake above nonspe-

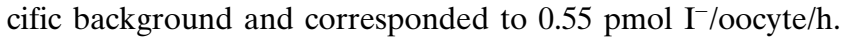
As reported previously (17), total mRNA had no detectable $\mathrm{I}^{-}$ transport activity.

The paternal allele. The entire coding region of the proposita's NIS was sequenced directly from cDNA transcribed from thyroid tissue RNA. A single nt substitution, $C$ to $\mathrm{G}$ transversion at nt 1146, was found in exon 6, which replaces the normal Gln (CAG) in codon 267 with a Glu (GAG) (Q267E). The proposita appeared to be homozygous for this mutation since the WT nt $1146 \mathrm{C}$ was not detected (Fig. 3 B). However, the genomic sequence of the corresponding region indicated that the

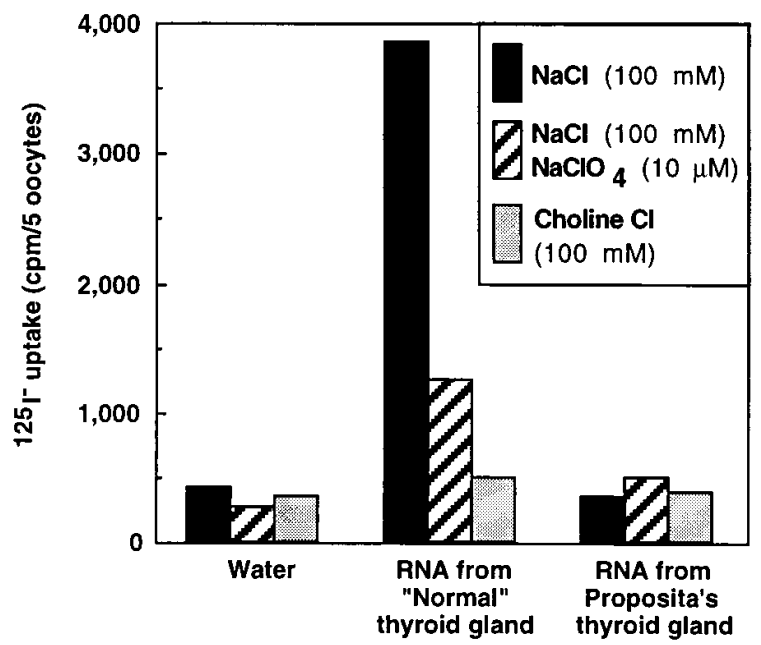

Figure 2. Activity of NIS measured by translation of thyroid gland mRNA in Xenopus oocytes. Size-selected (2-4.5 kb) polyA ${ }^{+}$RNA from a multinodular thyroid gland ("Normal") and from the thyroid gland of the proposita were injected into oocytes, and $\mathrm{I}^{-}$uptake was measured under three different conditions, as described in Methods. Note the lack of specific, $\mathrm{NaClO}_{4}$-sensitive $\mathrm{I}^{-}$uptake in oocytes injected with the proposita's RNA.

patient was heterozygous for this mutation (Fig. $3 A$ ). Furthermore, genotyping for this mutation showed that the mutation was inherited from the father and that it was also present in the unaffected brother and in a paternal uncle and aunt (Fig. $1 B$ ). The unaffected mother, sister, and three maternal uncles, as well as 50 normal subjects, had only the WT nt $1146 \mathrm{C}$.

DNA fragments encompassing nt 1146 were amplified by PCR from eight different cDNAs transcribed from three different RNA preparations, extracted from different areas of the proposita's thyroid gland. They were used to prepare clones in pGEM-T vector. Each of the 28 randomly selected clones harbored the mutant nt $1146 \mathrm{G}$. The same result was obtained when cDNA was generated by illegitimate transcription of NIS from lymphocyte RNA (10 of 10 clones). In contrast, illegitimate transcription of NIS from lymphocyte RNA of the unaffected, heterozygous brother yielded five clones with the WT nt $1146 \mathrm{C}$ and six with the mutant nt $1146 \mathrm{G}$.

The mutant NIS 267E was expressed in COS-7 cells, and its $\mathrm{I}^{-}$transport activity was compared to that of the WT NIS under the same conditions (Fig. 4). Results of $\mathrm{I}^{-}$uptake were corrected for the efficiency of transfection using a cotransfected luciferase expression vector, and were corrected for nonspecific retention of ${ }^{125} \mathrm{I}^{-}$on plastic by subtracting the counts per minute retained in empty wells processed in parallel. $\mathrm{ClO}_{4}^{-}-$ sensitive $\mathrm{I}^{-}$uptake in cells transfected with the WT NIS (Q267) was 24-fold above the choline background. The mutant NIS 267E showed no significant $\mathrm{I}^{-}$uptake above the choline background and was not different from the $\mathrm{I}^{-}$uptake of cells transfected with the empty vector serving as a negative control. Furthermore, cotransfection of equal amounts of WT and mutant NIS did not reduce the $\mathrm{I}^{-}$uptake activity of the WT NIS.

The maternal allele. The results shown in Fig. $1 B$ and Fig. $3, A$ and $B$, suggested that the maternal allele was either weakly expressed or not expressed at all. Therefore, the pro- 


\section{Paternal allele

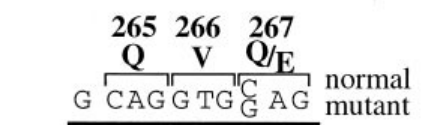 \\ A

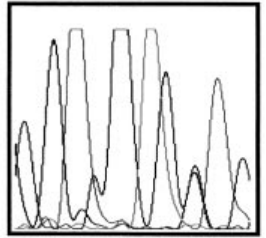 \\ heterozygous}

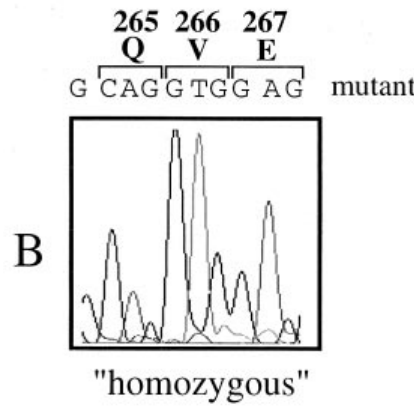

preferentially expressed
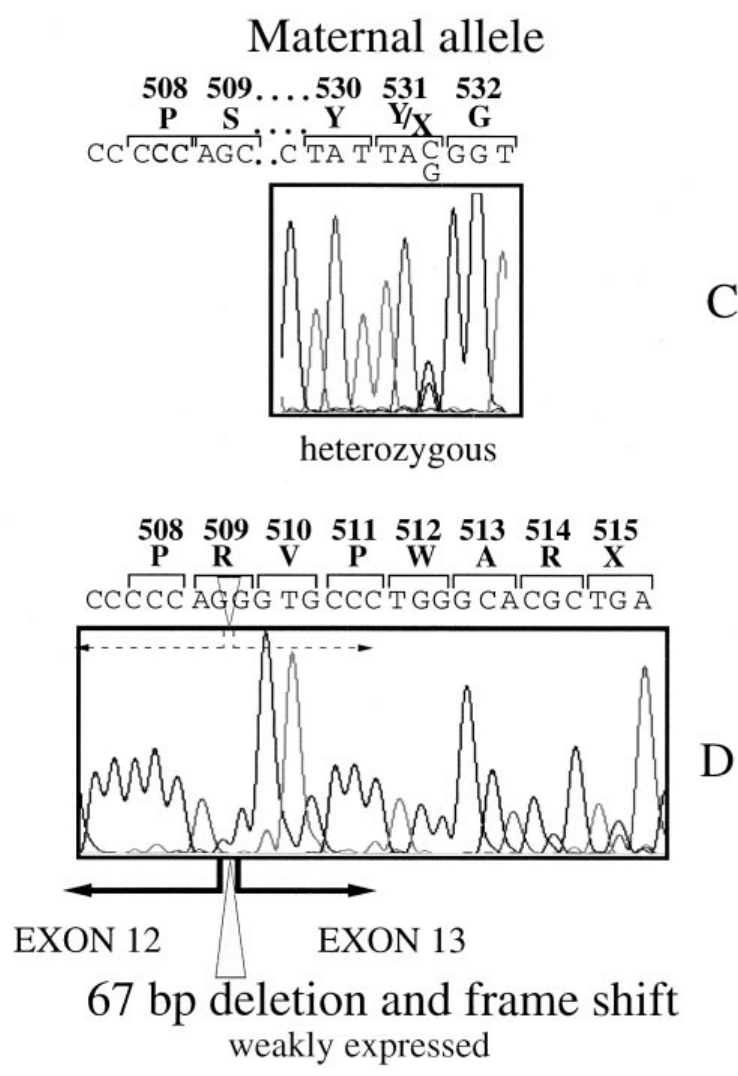

Figure 3. Sequences of

$\mathrm{C}$ the proposita's genomic

DNA and cDNA. De-

spite heterozygosity at the genomic level $(A)$, only the mutant paternal allele is visible by direct sequencing of cDNA $(B)$. This mutation produces a single amino acid substitution (Q267E). The mutation in the maternal allele, a $\mathrm{C} \rightarrow \mathrm{G}$ in codon $531(C)$, produces a 67-bp deletion in the cDNA, resulting in a frameshift and premature stop at codon 515 $(D)$. The latter could be demonstrated only after allele-specific amplification of cDNA, indicating a very low level of expression. posita's NIS cDNA was amplified using primers specific for the WT nt 1146C that according to genotyping should be present. The two cDNA fragments thus generated in both directions and starting at nt $1146 \mathrm{C}$ (see Methods) were ligated

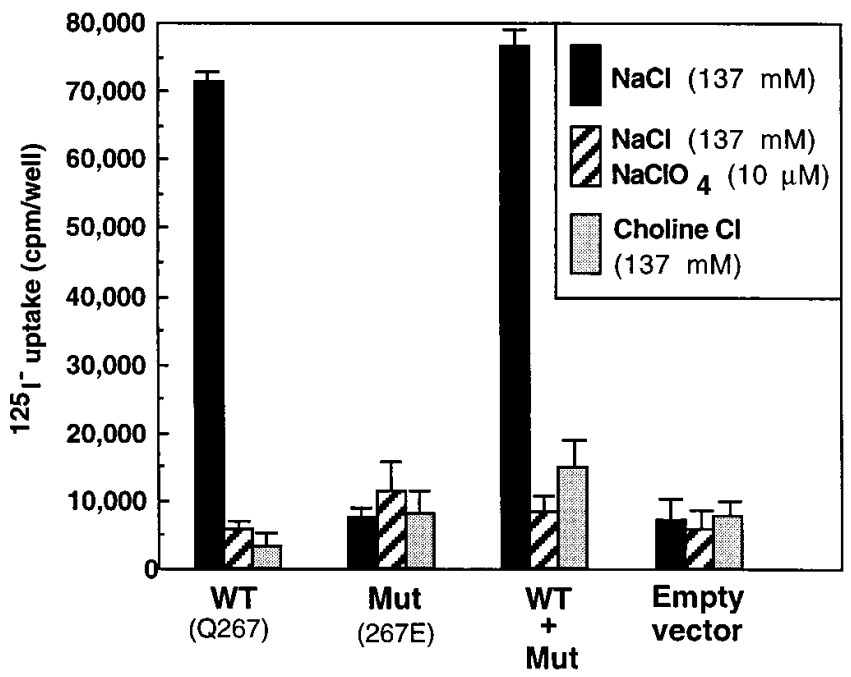

Figure 4. Functional analysis of the mutant NIS Q267E. Expression plasmids of the WT NIS Q267 and the mutant (Mut) NIS 267E were transfected into COS-7 cells alone, and together in equal amounts $(W T+M u t)$. Empty vector was used as a control. Results were corrected for the efficiency of transfection by cotransfection of a luciferase-expressing plasmid and reported in this representative experiment as mean \pm range of duplicate determinations. Specific, $\mathrm{NaClO}_{4^{-}}$ sensitive $\mathrm{I}^{-}$uptake was not observed with the mutant NIS (267E). Furthermore, the presence of the mutant NIS did not inhibit the activity of the WT NIS $(W T+M u t)$. into pGEM-T and sequenced. In the $3^{\prime}$ direction, seven of eight clones displayed a 67-bp deletion (nt 1874-1940) causing a frameshift and a premature stop at codon 515 (Fig. 3 D). The resulting protein would lack the 129 carboxy-terminal amino acids.

Sequencing genomic DNA of the proposita provided the explanation for this deletion. The proposita was heterozygous for a $\mathrm{C}$ to $\mathrm{G}$ transversion in $\mathrm{nt} 1940$ located in exon 13 (Fig. 3 $C$ ). While this nt substitution produces a stop (TAG) at codon 531, it also creates a new $3^{\prime}$ splice acceptor site (AC to AG) for intron 12 (14), located downstream of the authentic site. This new cryptic $3^{\prime}$ splice acceptor site with its corresponding branch site matches the mammalian consensus sequences (Fig. 5) better and is therefore used preferentially. The mutation in nt 1940 was also found in the unaffected mother, sister, and in one of three uncles, but not in the father or brother (Fig. 1C).

The one of eight pGEM-T clones containing NIS cDNA from the proposita that did not have the 67-bp deletion contained, nevertheless, the mutation in nt 1940. This indicates that the cryptic $3^{\prime}$ splice is not used exclusively.

\section{Discussion}

We describe two novel mutations of the NIS gene that cause congenital hypothyroidism in a compound heterozygous individual. While both mutations involve single base pair substitutions in coding regions, one of the two is unique. It is located in an exon creating a new downstream cryptic $3^{\prime}$ splice acceptor site by introducing a $\mathrm{G}$ at position -1 of this site.

The mutation (Q267E), inherited from the father, is located in the fourth extracellular loop between the seventh and eighth segments of the transmembrane domain, as deduced 


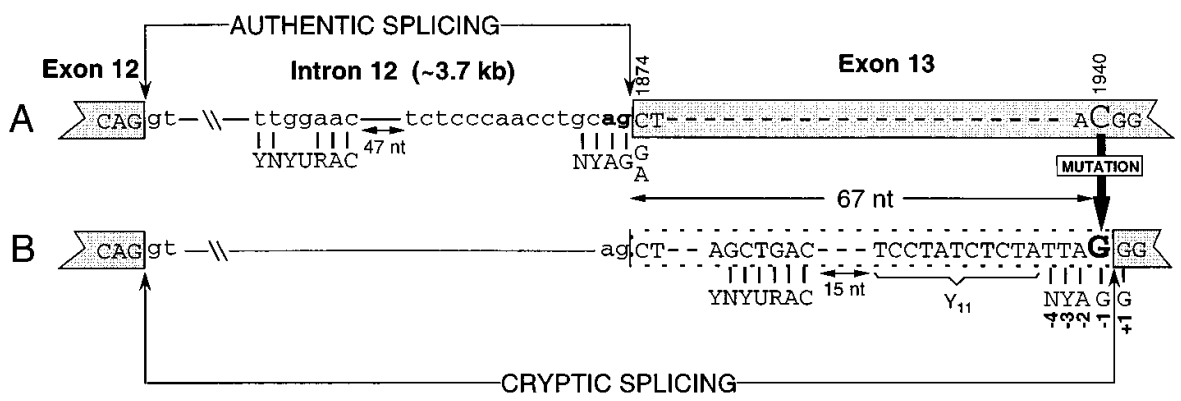

Figure 5. Comparison of authentic $(A)$ and cryptic $(B)$ splicing of intron 12 . The maternal mutation $\mathrm{C} 1940 \rightarrow \mathrm{G}$ (thick arrow and large bold letter) creates a new $3^{\prime}$ acceptor splice site that results in a 67-nt deletion in the cDNA of exon 13. Matched nt to the consensus sequences for branch sites (YNYURAC) and for the 3' splice acceptor sites $\left(\mathrm{NYAG}^{\mathrm{G}} / \mathrm{A}\right)$ are indicated (vertical lines), but not for the 11-nt polypyrimidine tracts $\left(\mathrm{Y}_{11}\right)$. The position number of $\mathrm{nt}$ at the $3^{\prime}$ splice site is indicated. Polypyrimi-

dine tract sequences of both the authentic and cryptic $3^{\prime}$ acceptor splice sites are mismatched in two adenines. In contrast, there is an additional $\mathrm{nt}$ mismatch in both the branch and acceptor sites of the authentic compared with the cryptic splicing sequence. The number of nt between the branch and acceptor sites is indicated. $Y$, Pyrimidine. $R$, Purine. $N$, Any nt. $U$, Uracil.

from the hydropathic and secondary structure of the protein (12). Compared to the 12-segment model for the transmembrane domain of other sodium-dependent cotransporters (23), it lies close to one of the two described "hot spot" areas in the human SGLT1 (24). Recently, Western blot analysis has shown that a missense mutation from Cys to Ser in codon 355 (C355S) in the SGLT1 gene, located similarly in the fourth extracellular loop of the predicted $S G L T 1$ protein, results in a nonfunctional protein by blocking its transfer from the endoplasmic reticulum to the cell membrane (25). This mechanism could be the underlying defect in the mutant NIS Q267E and will be investigated as soon as an antibody against the extracellular domain of NIS becomes available. Provided the mutant protein is expressed properly in the membrane, an exchange of a neutral Gln for a negatively charged Glu could alter the conformation of the fourth extracellular loop, resulting in an impairment of function.

The functional property of mutant $267 \mathrm{E}$ was examined by expression in COS-7 cells. It did not demonstrate detectable $\mathrm{I}^{-}$ transport activity. However, based on the coefficient of variation of the $\mathrm{I}^{-}$uptake assay of $3 \%$ for the WT NIS, some activity below this level cannot be excluded. Cotransfection of equal amounts of WT NIS and mutant NIS 267E resulted in a normal $\mathrm{I}^{-}$uptake. These data are in agreement with the clinical observation that heterozygotes for NIS Q267E are clinically normal, provided they express one normal allele. This held true for both mutations (Fig. $1 A$ ).

A patient with $\mathrm{I}^{-}$transport defect, homozygous for a mutation (T354P) located in the ninth segment of the transmembrane domain, was reported very recently (10). The mutant NIS 354P had no detectable $\mathrm{I}^{-}$transport activity in HEK-293 cells.

In our case, the second point mutation, inherited from the mother and also a $\mathrm{C}$ to $\mathrm{G}$ transversion, is located in codon 531 of exon 13. Although this mutation predicts a stop at codon 531 (TAC $\rightarrow$ TAG), it also creates a new $3^{\prime}$ splice acceptor site (AC to AG) for intron 12 that is $67 \mathrm{nt}$ downstream of the authentic site. It leads to the deletion of 67 bases from the mRNA transcript. This deletion results in a frameshift and a premature stop at codon 515 (Fig. $3 \mathrm{D}$ ). A protein translated from such a transcript would lack the entire twelfth segment of the transmembrane domain, and is therefore predicted to be biologically inactive. However, cDNA from thyroid and lymphocytes of the proposita appeared to be homozygous for the paternal mutant allele, NIS 267E (Fig. 3 B), and only allele- specific primers enabled us to amplify the maternal allele. Since none of the 38 cDNA clones prepared from the proposita's tissues (28 from thyroid and 10 from lymphocytes) were transcribed from the maternal allele, it can be calculated that expression of the corresponding mature transcript is very low $(<2.5 \%)$. It is well known that eukaryotic cells have mechanisms to degrade RNAs that harbor premature termination codons, presumably for protection against the formation of abnormal proteins that could endanger cell viability (26). This explains the low steady state level of the mutant mRNA that could be demonstrated only after allele-specific amplification of the cDNA.

We showed that the small amount of NIS mRNA transcribed from the maternal allele was more abundant in the cryptic spliced form. Of eight NIS cDNA clones generated by specific amplification of the maternal allele, seven displayed the 67-nt deletion, and one was full-length with the mutation in exon 13. Taking into account that the maternal allele is represented in $<2.5 \%$ of the total NIS mRNA (see above), and that only one of eight clones is derived from properly spliced NIS mRNA of this allele, it can be calculated that the latter accounts for $<0.3 \%$ of the total NIS mRNA of the proposita. However, this cannot explain the very low $\mathrm{I}^{-}$concentration in thyroid and salivary gland observed in the proposita, because the mutation produces a stop irrespective of the site of splicing.

Splice site mutations are not uncommon. They represent $15 \%$ of all point mutations causing human genetic diseases (27). More than two thirds affect 5' splice donor sites and usually result in exon skipping. Preferential use of a cryptic splice site over the authentic site is less common, particularly when it involves the AG consensus sequence of the $3^{\prime}$ splice site. When aberrant splicing creates a new $3^{\prime}$ splice acceptor site, the latter is usually located upstream of the authentic $3^{\prime}$ splice site, thus adding intronic sequences to the downstream exon (27, 28). Downstream exonic cryptic 3 ' splice acceptor sites have been reported in four instances, and in three of them, the mutations were located in the new polypyrimidine tract (2932). Only one of these mutations, recently described, created a cryptic $3^{\prime}$ splice acceptor site motif used preferentially over the upstream authentic splice site (32). It introduced an A at position -2 . It is of interest that this position and not the introduction of a $\mathrm{G}$ in position -1 is responsible for all mutations creating novel $3^{\prime}$ acceptor splice sites irrespective of their location relative to the authentic site (27). 
In our case, replacement of the normal $\mathrm{C}$ for a $\mathrm{G}$ is unique, since it introduces a $\mathrm{G}$ at position -1 to create a new $3^{\prime}$ splice acceptor site downstream of the authentic splice site. These two sites, including their corresponding polypyrimidine tracts and branch sites, are compared in Fig. 5. To determine what component of the splice acceptor site recognition may be responsible for the preferential use of the cryptic site, the consensus values were calculated according to the scoring system of Shapiro and Senapathy (33). In this system, nt at each position of the splice complex are scored for their frequency. A splice site containing the most frequent bases at each position would have a consensus value of 1 , while that with the least frequent bases would yield a consensus value of 0 . Thus, in comparing the consensus values of cryptic and authentic $3^{\prime}$ splice sequences, a higher value identifies the sequence responsible for the successful use of a new splice site and its competition with the authentic site. The consensus values for the cryptic and authentic splice sequences, respectively, are as follows: 0.818 and 0.868 for the five $n t(+1$ to -4$)$ representing the $3^{\prime}$ splice acceptor site proper, and 0.588 and 0.595 for the polypyrimidine tract. These scores suggest that the authentic splice site matches the consensus sequence better than the cryptic site even though there is a mismatch at position +1 of the authentic site. In contrast, the branch site of the cryptic splice site matches the consensus sequence more closely than the corresponding authentic site. Unfortunately, no nt frequency data are available to calculate a consensus value score for the branch site. Since our results show that the cryptic $3^{\prime}$ acceptor splice site is used preferentially, we conclude that the new branch site sequence and its closer proximity to the polypyrimidine tract are likely responsible for the activation of this site in the maternal allele.

To reconcile the genetic data with the phenotype, possible explanations for the apparent low level of $\mathrm{I}^{-}$concentrating activity observed in vivo have been considered. The increased vascularity of the hyperplastic thyroid gland could explain the $3 \%$ thyroidal $\mathrm{I}^{-}$uptake measured at $2 \mathrm{~h}$, after the standard subtraction of background counts obtained over the thigh. The loss of detectable thyroidal $\mathrm{I}^{-}$uptake at 6 and $24 \mathrm{~h}$ supports this interpretation, though reduced activity in the right lower pole mass, which appeared to be highly vascular by ultrasound, does not. The S/P I ${ }^{-}$ratio of 2.5 rather than 1 indicates a low level of $\mathrm{I}^{-}$concentration in a tissue that actively transports $\mathrm{I}^{-}$. However, since the radioiodide was given by mouth rather than intravenously, we cannot exclude a low level of residual contamination from the administered dose. Since the mutant NIS inherited from the mother would be nonfunctional even if it were expressed, a low level of $\mathrm{I}^{-}$concentrating activity of the paternal mutant NIS $267 \mathrm{E}$ is an explanation for the apparent $\mathrm{I}^{-}$transport activity observed in vivo. Although no $\mathrm{I}^{-}$concentrating activity could be detected in COS-7 cells transfected with this mutant NIS, the limit of precision of the in vitro $\mathrm{I}^{-}$ uptake assay was $3 \%$, which is within the range of relative activity observed in vivo.

The low prevalence of $\mathrm{I}^{-}$transport defect as a cause of congenital hypothyroidism is not well understood. Though the frequency of mutations in the NIS gene is not known, impairment of thyroidal $\mathrm{I}^{-}$concentration is expected to occur only if both NIS alleles are nonfunctional. However, because under conditions of high iodide intake, full preservation of $\mathrm{I}^{-}$concentrating function is not required to achieve normal hormone synthesis, it can be speculated that only mutations in the NIS gene that cause a virtually complete loss of function will be detected.

\section{Acknowledgments}

The authors thank Dr. Kenneth B. Ain for providing human NIS sequences, and Dr. Graeme I. Bell for valuable advice and for his critical review of the manuscript.

This work is supported in part by grants from the National Institutes of Health (DK-15070 and RR-00055). J. Pohlenz is supported in part by a grant from the Deutsche Forschungsgemeinschaft (Po $556 / 1-1)$.

\section{References}

1. Dai, G., O. Levy, L.M. Amzel, and N. Carrasco. 1996. The mediator of thyroidal iodide accumulation: the sodium/iodide symporter. In Handbook of Biological Physics. Vol. 2. W.N. Konings, H.R. Kaback, and J.S. Lolkema, editors. Elsevier Science B.V., Amsterdam. 343-368.

2. Medeiros-Neto, G., and J.B. Stanbury. 1994. Inherited Disorders of the Thyroid System. CRC Press, Inc., Boca Raton, FL. pp. 1-221.

3. Toublanc, J. 1992. Comparison of epidemiological data on congenital hypothyroidism in Europe with those of other parts of the world. Horm. Res. (Basel). 38:230-235.

4. Grüters, A. 1992. Congenital hypothyroidism. Pediatr. Ann. 21:24-28.

5. Vassart, G., J.E. Dumont, and S. Refetoff. 1995. Thyroid disorders. In The Metabolic and Molecular Basis of Inherited Disease. Vol. 2. C.R. Scriver, A.L. Beaudet, W.S. Sly, and D. Valle, editors. McGraw-Hill Inc., New York. 2883-2928.

6. Hayashizaki, Y., Y. Hiraoka, K. Tatsumi, T. Hashimoto, J. Furuyama, K. Miyai, K. Nishijo, M. Matsuura, H. Kohno, A. Labbe, and K. Matsubara. 1990 Deoxyribonucleic acid analyses of five families with familial inherited thyroid stimulating hormone deficiency. J. Clin. Endocrinol. Metab. 71:792-796.

7. Abramowicz, M.J., L. Duprez, J. Parma, G. Vassart, and C. Heinrichs. 1997. Familial congenital hypothyroidism due to inactivating mutation of the thyrotropin receptor causing profound hypoplasia of the thyroid gland. J. Clin. Invest. 99:3018-3024.

8. Bikker, H., T. Vulsma, F. Baas, and J.J.M. de Vijlder. 1995. Identification of five novel inactivating mutations in the human thyroid peroxidase gene by denaturing gradient gel electrophoresis. Hum. Mutat. 6:9-16.

9. Medeiros-Neto, G., H.M. Targovnik, and G. Vassart. 1993. Defective thyroglobulin synthesis and secretion causing goiter and hypothyroidism. Endocr. Rev. 14:165-183.

10. Fujiwara, H., K. Tatsumi, K. Miki, T. Harada, K. Miyai, S.-I. Takai, and N. Amino. 1997. Congenital hypothyroidism caused by a mutation in the $\mathrm{Na}^{+} /$ $\mathrm{I}^{-}$symporter. Nat. Genet. 16:124-125.

11. Federman, D., J. Robbins, and J.E. Rall. 1958. Some observations on cretinism and its treatment. N. Engl. J. Med. 259:610-613.

12. Dai, G., O. Levy, and N. Carrasco. 1996. Cloning and characterization of the thyroid iodide transporter. Nature. 379:458-460.

13. Smanik, P.A., Q. Liu, T.L. Furminger, K. Ryu, S. Xing, E.L. Mazzaferri, and S.M. Jhiang. 1996. Cloning of the human sodium iodide symporter. Biochem. Biophys. Res. Commun. 226:339-345.

14. Smanik, P.A., K.-Y. Ryu, K.S. Theil, E.L. Mazzaferri, and S.M. Jhiang. 1997. Expression, exon-intron organization, and chromosome mapping of the human sodium iodide symporter. Endocrinology. 138:3555-3558.

15. Medeiros-Neto, G., W. Bloise, and A.B. Ulhoa-Cintra. 1972. Partial defect of iodide trapping mechanism in two siblings with congenital goiter and hypothyroidism. J. Clin. Endocrinol. Metab. 35:370-377.

16. Harden, R.M., W.D. Alexander, C.J.S. Chisholm, and J. Shimmins. 1968. The salivary iodide trap in nontoxic goiter. J. Clin. Endocrinol. Metab. 28 : 117-120.

17. Vilijn, F., and N. Carrasco. 1989. Expression of the thyroid sodium/iodide symporter in Xenopus laevis oocytes. J. Biol. Chem. 264:11901-11903.

18. Ishikawa, Y., K. Tokunaga, K. Kashiwase, T. Akaza, K. Tadokoro, and T. Juji. 1995. Sequence-based typing of HLA-A2 alleles using a primer with an extra base mismatch. Hum. Immunol. 42:315-318.

19. Weiss, R.E., M. Weinberg, and S. Refetoff. 1993. Identical mutations in unrelated families with generalized resistance to thyroid hormone occur in cytosine-guanine-rich areas of the thyroid hormone receptor beta gene. Analysis of 15 families. J. Clin. Invest. 91:2408-2415.

20. Hayashi, Y., T. Sunthornthepvarakul, and S. Refetoff. 1994. Mutations of $\mathrm{CpG}$ dinucleotides located in the triiodothyronine $\left(\mathrm{T}_{3}\right)$-binding domain of the thyroid hormone receptor (TR) $\beta$ gene that appears to be devoid of natural mutations may not be detected because they are unlikely to produce the clinical phenotype of resistance to thyroid hormone. J. Clin. Invest. 94:607-615.

21. Burant, C.F., and G.I. Bell. 1992. Mammalian facilitative glucose transporters: evidence for similar substrate recognition sites in functionally mono- 
meric proteins. Biochemistry. 31:10414-10420.

22. Weiss, S.J., N.J. Philp, and E.F. Grollman. 1984. Iodide transport in a continuous line of cultured cells from rat thyroid. Endocrinology. 114:10901098 .

23. Turk, E., C.J. Kerner, M.P. Lostao, and E.M. Wright. 1996. Membrane topology of the $\mathrm{Na}^{+} /$glucose cotransporter SGLT1. J. Biol. Chem. 271:19251934.

24. Martin, M.G., E. Turk, M.P. Lostao, C. Kerner, and E.M. Wright. 1996. Defects in sodium/glucose cotransporter (SGLT1) trafficking and function cause glucose malabsorption. Nat. Genet. 12:216-220.

25. Martin, M.G., M.P. Lostao, E. Turk, J. Lam, M. Kreman, and E.M. Wright. 1997. Compound missense mutations in the sodium/D-glucose cotransporter result in trafficking defects. Gastroenterology. 112:1206-1212.

26. Maquat, L.E. 1996. Defects in RNA splicing and the consequence of shortened translational reading frames. Am. J. Hum. Genet. 59:279-286.

27. Cooper, D.N., and M. Krawczak. 1995. Human Gene Mutations. BIOS Scientific Publishers Ltd., Oxford. 412 pp.

28. Nakai, K., and H. Sakamoto. 1994. Construction of a novel database containing aberrant splicing mutations of mammalian genes. Gene (Amst.). 141:
$171-177$.

29. Wakamatsu, N., H. Kobayashi, T. Miyatake, and S. Tsuji. 1992. A novel exon mutation in the human $\beta$-hexosaminidase $\beta$ subunit gene affects $3^{\prime}$ splice site selection. J. Biol. Chem. 267:2406-2413.

30. Hasegawa, Y., H. Kawame, H. Ida, T. Ohashi, and Y. Eto. 1994. Single exon mutation in arylsulfatase A gene has two effects: loss of enzyme activity and aberrant splicing. Hum. Genet. 93:415-420.

31. Kajihara, C., S. Matsuhashi, K. Yamamoto, K. Kido, K. Tsujii, A Tanae, S. Fujiyama, T. Itoh, K. Tanigawa, M. Uchida, et al. 1995. Exon redefinition by a point mutation within exon 5 of the glucose-6-phosphatase gene is the major cause of glycogen storage disease type Ia in Japan. Am. J. Hum. Genet. 57:549-555.

32. Pompinio, R.J., T.R. Reynolds, H. Mandel, O. Admoni, P.D. Melone, G.A. Buck, and B. Wolf. 1997. Profound biotinidase deficiency caused by a point mutation that creates a downstream cryptic $3^{\prime}$ splice acceptor site within an exon of the human biotinidase gene. Hum. Mol. Genet. 6:739-745.

33. Shapiro, M.B., and P. Senapathy. 1987. RNA splice junctions of different classes of eukaryotes: sequence statistics and functional implications in gene expression. Nucleic Acids Res. 15:7155-7174. 